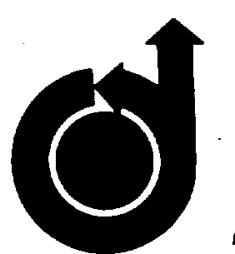

AIAA 76-374

Investigation of Turbulent Boundary-Layer Separation Using Laser Velocimetry D. Modarress and D. A. Johnson 


\title{
Investigation of Turbulent Boundary-Layer Separation Using Laser Velocimetry
}

\author{
D. Modarress* and D. A. Johnson $\dagger$ \\ NASA Ames Research Center, Moffett Field, Calif.
}

\begin{abstract}
Boundary-layer measurements realized by laser velocimetry are presented for a Mach 2.9, two-dimensional, shock-wave/lurbulent boundary-layer interaction containing an extensive region of separated flow. Mean velocity and Iurbulent intensity profiles were obtained from upstream of the interaction zone to downstream of the mean realtachment point. The superiority of the laser velocimeter technique over pressure sensors in turbulent separated flows is demonstrated by a comparison of the laser velocimeter data with results obtained from local pitot and static pressure measurements for the same flow conditions. The locations of the mean separation and reattachment points as deduced from the mean velocity measurements are compared to oil-flow visualization results. Representative velocity probability density functions obtained in the separated flow region are also presented. Critical to the success of this invesligation were 1) the use of Bragg cell frequency shifting and 2) artificial seeding of the flow with submicron light-scattering particles.
\end{abstract}

\section{Introduction}

$\mathbf{U}$ NTIL recently, numerical solution of the time-averaged conservation equations for the separated flow produced by a shock wave impinging on a turbulent boundary layer was beyond the scope of computational fluid dynamics. Now, with the development of better numerical codes and more advanced computers, a number of investigators have made predictions of this type of flow. ${ }^{1.5}$ However, still open to question is how satisfactorily the turbulence transport properties are being modeled. To test the various turbulence models employed, comparisons with experiments are needed. Unfort unately, the only mean velocity profile data available for comparison have been those obtained with pitot and static pressure probes, which must be considered suspect within and near the separation region because of the very high turbulence levels present. The degree of turbulence is such that, over a large portion of the flow, the velocity component in the streamwise direction fluctuates in sign. Thus, regardless of whether the pressure probes are aligned with the freestream flow or faced in the backward direction, measurement errors will result. Also of concern are the flow disturbances produced by the probes themselves.

The laser velocimeter, because of its nonintrusive nature, presents an attractive alternative in the study of separated flows. Its importance in general fluid flow research is well recognized. ${ }^{6}$ The advantages of laser velocimetry over conventional instrumentation become more evident as the complexity of the flows increase. For example, in the study of compressible turbulent flows, the Reynolds normal and shear stresses can be measured without the signal interpretation difficulties involved with hot-wire anemometry. In regions of turbulent separation, forward and reverse instantaneous velocities can easily be distinguished through frequency shifting techniques. Such directional information is not achievable with other existing techniques. Also, since it has a truly linear response, no inaccuracies need result when the fluctuations are large compared to the mean value.

Presented as Paper 76-374 at the AIAA 9h Fluid and Plasma Dynamics Conference, San Diego, Calif., July 14-16, 1976; submitted Aug. 12, 1976; revision received Jan. 14, 1979. Copyright (C) American Institute of Aeronautics and Astronautics, Inc., 1976. All rights reserved.

Index categories: Lasers; Boundary Layers and Convective Heal Transfer-Turbulent.

- NRC Research Associate. Presently Assistant Professor, ARYAMEHR University of Technology, Tehran, Iran. Member AIAA.

+Research Scientist. Member AlAA.
Several investigators have demonstrated that the laser velocimeter can provide accurate localized velocity information in high-speed wind tunnels. Favorable mean velocity comparisons with pitot-tube measurements have been obtained for turbulent supersonic boundary layers with zero pressure gradient. ${ }^{7.9}$ The Reynolds normal and shear stresses have been measured for an undisturbed turbulent boundary layer ${ }^{9,10}$ and for a relatively mild (unseparated case) shockwave/turbulent boundary-layer interaction. "For the points away from the wall, fluctuation measurements agreed well with hot-wire anemometer measurements.

The major shortcoming of the laser velocimeter technique is that minute particles are required in the detection process. These particles must be large enough to provide the scattered light levels required by the detection electronics and yet small enough to follow the fluid motion. In compressible flows, the particle trackability requirements can be especially extreme because of the existence of large spatial velocity gradients and/or high, turbulent convection velocities. However, the need for a better understanding of the behavior of these flows and the potentials of laser velocimetry provide the impetus to overcome this difficulty.

Another controversial problem associated with laser velocimetry is the error associated with the velocity biasing. In Ref. 12 it is argued that the particle passage through the sensing volume is not independent of the instantaneous velocity field. The probability of a particle occurrence is directly proportional to the magnitude of the instantaneous velocity vector $\left|v_{i}\right|$, and moreover, this weighting function is independent of the concentration of particles. Hence, any meaningful correction to the readings is done when $\left|v_{i}\right|$ is known. Experimental results have shown that to correct the dat a on the basis of a one-dimensional model would result in an overcorrection and more erroneous results.

In the present investigation, a directionally sensitive laser velocimeter system was used to study the interaction of an externally generated oblique shock wave and a turbulent boundary layer for a freestream Mach number of 2.9. The interaction was sufficiently strong to produce an extensive region of recirculating flow. Mean velocities and turbulence intensities in the streamwise direction were obtained throughout this interaction region with the velocimeter system. Representative examples of these mean velocity profiles are compared to results obtained with pitot and static pressure probes for the same flow conditions. The approximate locations of the mean separation and reattachment points as determined from the velocity measurements are 


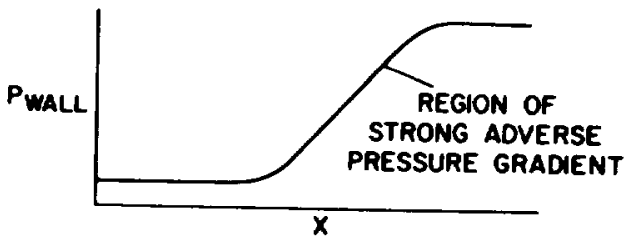

NOZZLE WALL BOUNDARY LAYER
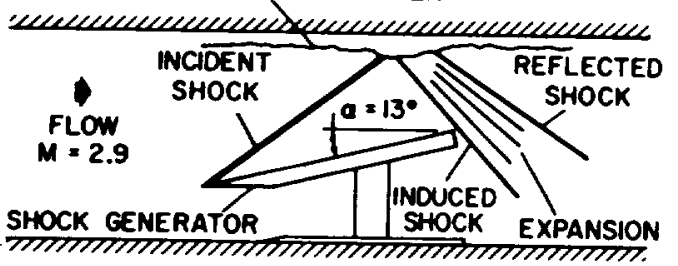

Fig. 1 Flow model for shock-induced separation.

compared to oil-flow visualization results. Representative velocity probability density functions are presented to show the difference in the character of the flow near the separation and reattachment points.

\section{Experimental Apparatus}

The investigation was conducted in the Ames $8 \times 8$ in. supersonic wind tunnel. A full-span wedge set at an angle of $13 \mathrm{deg}$ with respect to the oncoming flow was used to generate the oblique shock wave which impinged on the upper nozzle wall boundary layer, thereby causing separation. A schematic of a flow model is shown in Fig. 1. The freestream Mach number was 2.9 with a unit Reynolds number of $5.7 \times 10^{7} / \mathrm{m}$. The initial boundary-layer thickness $\delta_{0}$ was $1.7 \mathrm{~cm}$, and the pressure rise produced by the shock wave was sufficiently strong $\left(p_{\text {inal }} / p_{\text {initial }}=5\right)$ to cause a separation region nearly 6 $\mathrm{cm}$ long. The flowfield produced by this model has been previously investigated by Reda and Murphy. ${ }^{13}$ In that study, surface pressures, Schlieren photographs, oil-flow visualizations, and local pitot and static pressures were obtained. Both in Ref. 13 and in this investigation adiabatic wall was assumed. The effects of side wall boundary-layers were studied by Reda and Murphy. ${ }^{14}$

The laser velocimeter system and its orientation to the supersonic tunnel are shown in a plan view in Fig. 2. It is a single-velocity component, "fringe" mode system with offaxis, forward-scatter light collection. The incoming beams were aligned perpendicular to the tunnel centerline with the fringes oriented for sensitivity to only the streamwise velocity component. To achieve a frequency shift, a solid crystal Bragg cell was used (the frequency shift was $40 \mathrm{MHz}$ ); this also accomplished the splitting of the original laser beam into two equal intensity beams.

The frequency-shifted and unshifted beams were brough parallel to each other by an optical cube that had been designed to compensate for the Bragg angle $\Omega$. The effective sensing volume of the velocimeter was approximately a cylinder $(0.3 \mathrm{~mm}$ in diameter and $1.5-\mathrm{mm}$ long) whose axis was in the cross-stream direction.

Single-particle, counter-type signal processing was used to measure the periods $\tau_{i}$ of the signal bursts produced by individual particles passing through the sensing volume. Histograms of these individual period realizations were developed with a multichannel analyzer that was, in turn, interfaced to a programmable calculator for on-line data reduction. Given this period information, the distribution of the streamwise velocity component $u$ was determined from the expression

$$
\frac{I}{\tau}=f= \pm f_{B}+\frac{1 / 2 \sin (\theta / 2)}{\lambda} u
$$

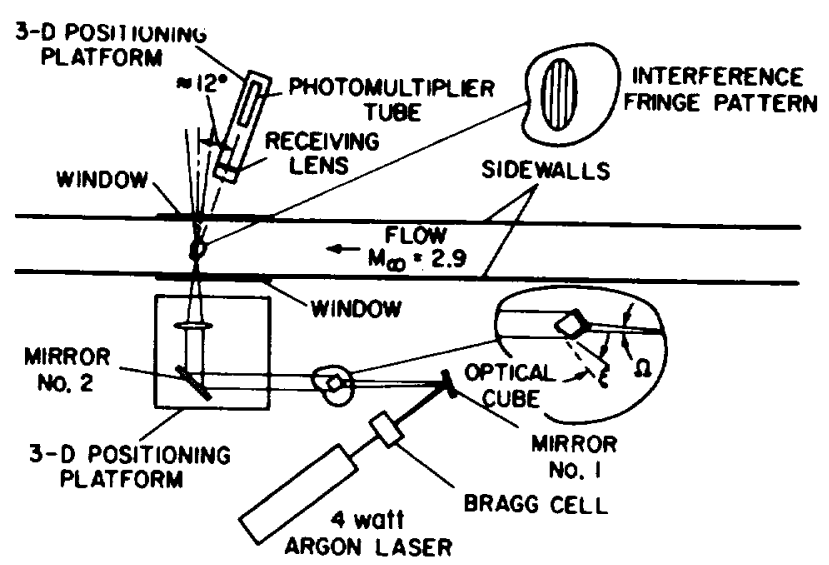

Fig. 2 Laser velocimeter system.

where $f$ is the frequency of the signal, $f_{B}$ the Bragg cell frequency shift (the sign depends on which incident beam is frequency shifted), $\lambda$ the wavelength of the laser light, and $\theta$ the angle between the two incident beams. Estimates of the mean rms velocities were realized from the statistical estimator

$$
\bar{u}=\frac{I}{N} \sum_{i=1}^{N} u_{i}
$$

and

$$
\left\langle u^{\prime}\right\rangle=\left(\overline{u^{\prime 2}}\right)^{1 / 2} \approx \frac{l}{N}\left[\sum_{i=1}^{N}\left(u_{i}-\bar{u}\right)^{2}\right]^{1 / 2}
$$

respectively, where $N$ is the total number of realizations.

Equations (2) and (3) are valid if the sampling is random and independent of the velocity being sensed. The velocity biasing error is directly proportional to the intensity of turbulence presented at the measurement point. Therefore, for highly turbulent flow regions such as those investigated in this st udy, the problem is more severe. To correct the data on the basis of the one-dimensional correction proposed in Ref. 12 would have produced overcorrected results and for the points with $u_{i}=0$ would have resulted in completely unreasonable results because of an inherent singularity of these points. In the present investigation, measurement for the streamwise component of the velocity vector were made. Therefore, it was decided to present the data in their uncorrected form.

Based on simple statistical considerations, the number of realizations needed to obtain accurate estimates of $\dot{u}$ and $\left\langle u^{\prime}\right\rangle$ increases with the level of turbulence. In the inner part of the boundary layer where the turbulence levels were high, 10 to 20 thousand realizations were obtained at each mesurement station. The effects of spurious readings due to low signal-tonoise ratio (SNR) conditions were reduced by ignoring velocity readings whose occurrences were more than a factor of 50 less likely than the most probable velocity.

In the freestream, the measured turbulence levels were higher than the expected values for the wind tunnel. To examine the effect of Bragg cell on the measured rms, measurement for identical flow conditions were made with and without Bragg cell frequency shifting. Theoretically, in the case of no frequency shift, from Eq. (1) we have

$$
f=\frac{(1 / 2) \sin (\theta / 2)}{\lambda} u
$$

hence, the relative turbulence intensity $\left\langle u^{\prime}\right\rangle / \bar{u}$ should be given by $\left\langle f^{\prime}\right\rangle / \bar{f}$. However, with Bragg cell frequency shifting, from Eq. (1), $\left\langle u^{\prime}\right\rangle / \bar{u}$ should be given by

$$
\left\langle u^{\prime}\right\rangle / \bar{u}=\left(f^{\prime}\right) /\left(\bar{f}-f_{B}\right)
$$




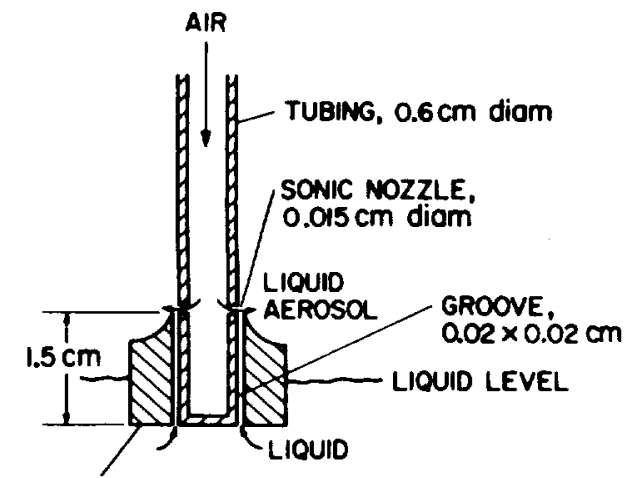

SLEEVE, $2 \mathrm{~cm}$ diam

Fig. 3 Laskin nozzle arrangement for aerosol generation.

Thus, for identical flow conditions, the difference in the two sets of measured rms values would indicate the effect of the Bragg cell on the measured rms. Experimental results showed no significant change in the rms for the two cases. The observed behavior implies that the excursions in the measured frequencies $\left\langle V^{\prime}\right\rangle$ in the freestream were predominat ely due to uncertainties in the measurements caused by noise-in-signal effects rather than by turbulent fluctuations.

If it is assumed that this signal-broadening effect due to noise is statistically independent of the turbulent fluctuations, it can be taken into account in the same manner as "Doppler ambiguity" broadening in spectrum-analyzer measurements. For the case of statistical independence, the total variance $\left\langle f^{\prime}\right\rangle^{2}$, of the measurements is given by the sum of the individual variances

$$
\left\langle f^{\prime}\right\rangle^{2}=\left\langle f_{n}^{\prime}\right\rangle^{2}+\left\langle u^{\prime}\right\rangle^{2}\left(\frac{\sin (\theta / 2)}{\lambda}\right)^{2}
$$

where $\left\langle f_{n}^{\prime}\right\rangle^{2}$ is the variance due to measurement uncertainties and the last term on the right-hand side is the variance due to turbulent fluctuations. Equation (4) was used to correct the rms measurements obtained with Bragg cell frequency shifting. A value of $\left\langle f_{n}^{\prime}\right\rangle^{2}$ was selected that resulted in a conservative value of $2 \%$ for the relative freestream turbulence level, $\left\langle u^{\prime}\right\rangle / \bar{u}$ (the freestream turbulence was, most likely, less than this value). Since the sum of the squares is involved, this correction of the rms data quickly became negligible with increased turbulence in the boundary layer.

Rather than use the naturally occurring particles in the flow, as was done in Refs. 8, 10, and 11, the flow was artificially seeded with latex pigment particles of known size $(0.5-\mu \mathrm{m}$ diam). An aqueous solution of these particles is atomized with a Laskin-type nozzle to form a liquid aerosol containing the solid latex particles. With subsequent evaporation of the water, an aerosol of just solid latex particles remains. The solution is made sufficiently dilute so that the probability of more than one latex particle being contained in a liquid droplet is very low. Thus, when the droplets evaporate the formation of doublets or higher multiples is low compared to the number of singlets.

Figure 3 illustrates the Laskin-type nozzle arrangement used to atomize this suspension. The liquid level is evaluated inside the grooves to the vicinity of the nozzles by the pressure drop across the jet and also by capillary forces. With this arrangement, the need for a continuous liquid level control is eliminated. The aerosol generator can run continuously for an extensive period of time when a large bath of liquid is used.

To evaluate the response of these particles and, hence, verify their nominal size, measurements were taken across the incident oblique shock wave produced by the 13-deg wedge. The results of these measurements, with and without artificial seeding, are shown in Fig. 4. Throughout the boundary layer the particle occurrence rate was at least 50 times greater with

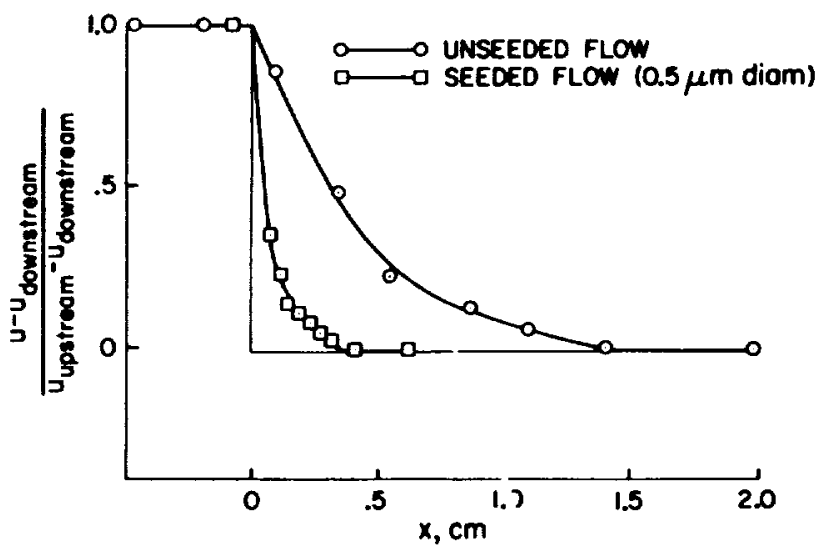

Fig. 4 Particle response to oblique shock wave at $M=2.9$ and $T_{t}=293 \mathrm{~K}$.

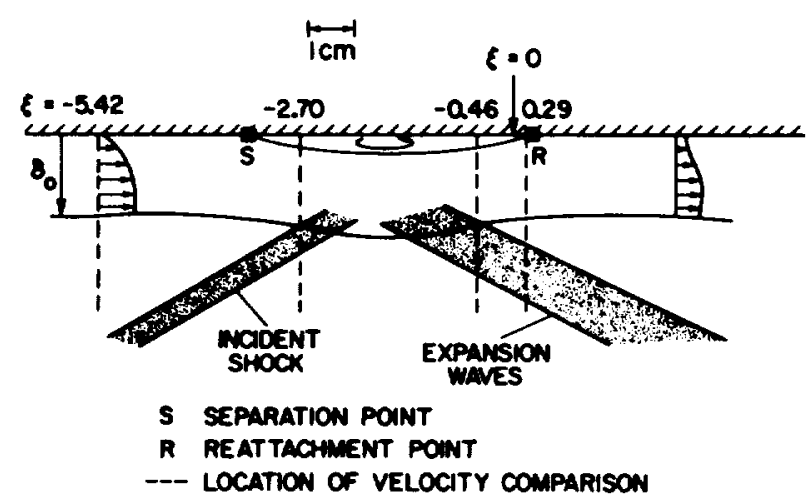

Fig. 5 Representation of flowfield generated by 13-deg wedge.

artificial seeding, so the presence of the natural particles had little effect on the response measurements of the seeded particles. In the realization of the boundary-layer measurements, the mean data rate was between 100 and $500 / \mathrm{s}$ with the seeded particles. The nominal relaxation distance (1/e point) of $0.2 \mathrm{~cm}$ of the latex particles corresponds to a frequency response of $50 \mathrm{kHz}$, which agrees well with that predicted from Stoke's drag law for a $0.5-\mu \mathrm{m}$ particle.

\section{Results}

The flowfield generated by the 13-deg wedge is schematically given in Fig. 5. The location of the incident shock wave and expansion fan were inferred from the Schlieren photographs of Ref. 13. The indicated width of the incident shock wave was caused by three dimensionalities of the flow and jitter in the shock wave. Laser velocimeter determinations of the mean velocity and turbulence intensity distributions across the turbulent boundary layer were made at 10 different streamwise stations. The most upstream measurement station was well upstream of the region of influence of the incident shock wave. The furthermost downstream station was approximately $4-\mathrm{cm}$ downstream of the reattachment point.

At some of these streamwise stations, local pitot and static pressures were available from Ref. 13 for comparison of mean velocity results. The results at four representative stations are presented in Figs. 6 and 7 . The location of these stations with respect to the interaction are designated in Fig. 5 . Distances are given in the nondimensional coordinate $\xi=\left(x-x_{i}\right) / \delta_{0}$, where $x_{i}$ is obtained by extrapolation of the incident shock wave to the wall.

In the upstream region of the interaction where the outer flow is parallel to the wall, the pressure data (pitot and static) should be valid, provided that there are no reverse velocities. In Fig. 6, the mean velocities obtained with the laser 

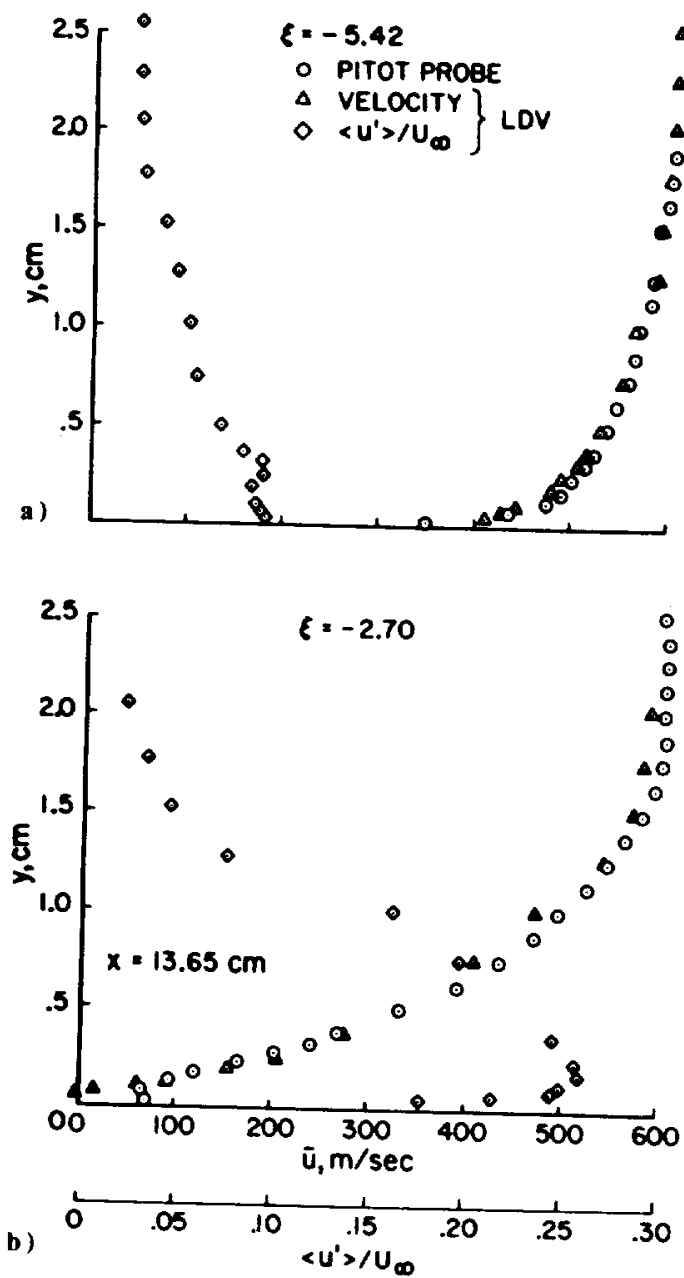

Fig. 6 Mean velocity comparison upstream of incident shock wave: a) upstream of interaction, b) near separation point.

velocimeter and pressure probes far upstream of the interaction (Fig. 6a) and near the separation point (Fig. 6b) are compared. The two sets of data are noted to be in good agreement, except at the wall near the separation point. At this location, the laser velocimeter indicated that there was backflow a high percentage of the time, which accounts for the overprediction in mean velocity with the pressure probes. The streamwise turbulence intensities relative to the freestream velocity $u_{\infty}$ as determined with the laser velocimeter, are also presented in Fig. 6. Notice the large change in turbulence levels between the two streamwise stations.

Profiles obtained in the separated flow region and near the boundary-layer reattachment are presented in Fig. 7. At these two stations, the measurements are seen to agree only in the middle portion of the boundary layer. The disagreement in boundary-layer edge velocities is believed to be caused by flow angularity outside the boundary layer, since, further downstream, both measurement techniques converge to the same edge velocity of $500 \mathrm{~m} / \mathrm{s}$. Near the wall, the laser velocimeter data show a behavior consistent with the physics of the flow; smooth profiles with negative mean velocities in the separated region and nonnegative mean velocities at reattachment. The pressure-probe data are obviously in error near the wall. Even if they were faced in the backward direction in the separated flow region, errors would be expected, as noted at the separation and reattachment points. At the other streamwise stations where pressure probe data were available, similar trends prevailed.

The complete mean velocity data obtained with the laser velocimeter are presented in Fig. 8, with the exception of the
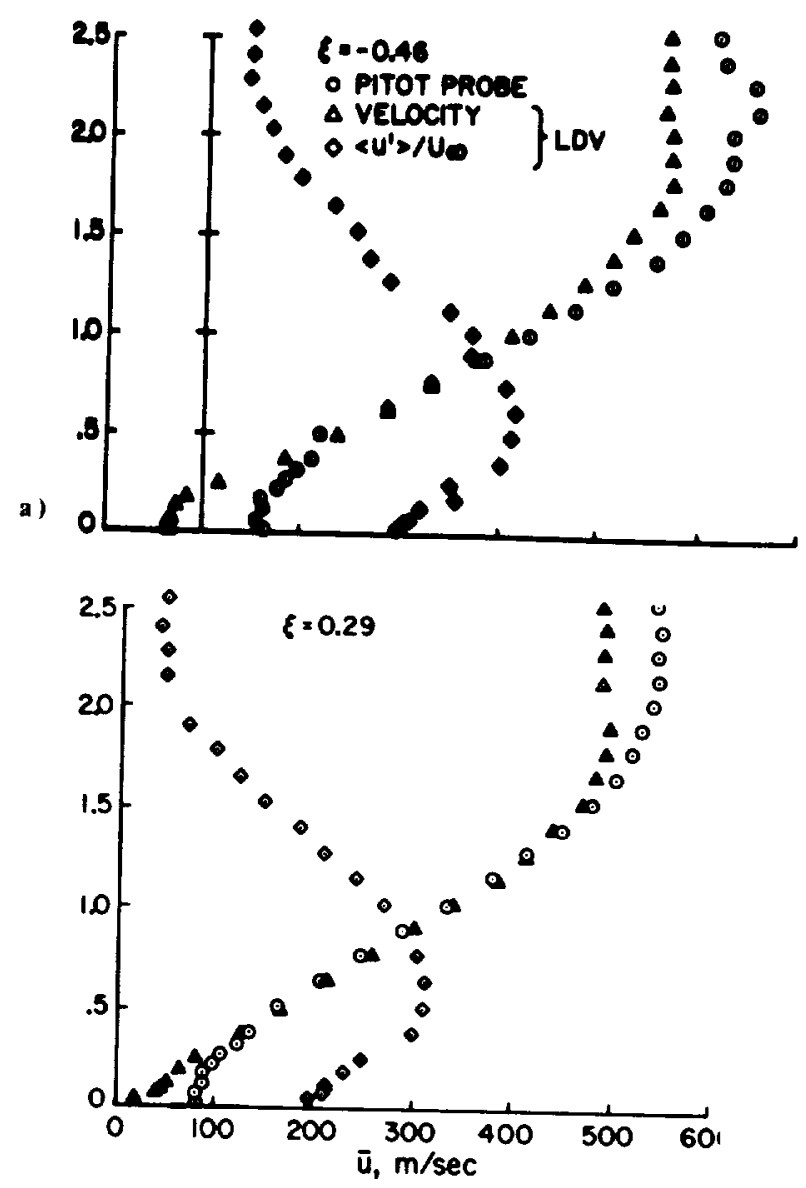

b)

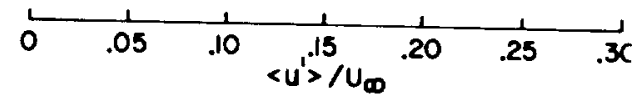

Fig. 7 Mean velocily comparison downstream of incident shock wave: a) within separated region, b) near reattachment point.

profile obtained far upstream of the interaction, $\xi=-5.42$. These profile data were taken at streamwise stations, $1.27-\mathrm{cm}$ apart $(\Delta \xi=0.75)$ in the interaction zone. At the station $\xi=-$ 4.21 , a very slight retardation of the flow near the wall was observed. This behavior is consistent with surface pressure measurements ${ }^{13}$ which indicate that the pressure at the wall starts to increase from the undisturbed value at $\xi=-4.3$. Further retardation near the wall is evident from the velocity profiles in the downstream station. The mean velocity profile at $\xi=-2.70$ clearly indicates separation; however it is not evident at the $\xi=-3.44$ station. According to the oil-flow visualization, ${ }^{13}$ mean separation occurs at $\xi=-3.41$. The discrepency in the determination of the actual point of separation may be explained by the fact that neither of the two methods can accurately locate the point of separation.

The oil-flow results could be misleading as the actual location of the separation and reattachment points since the oil buildup can influence and change these locations. $f$ On the other hand, the separated layer is exceptionally thin in this region (at $\xi=-2.70, \bar{u}=0$ at $y=0.5 \mathrm{~mm}$ ) and hence, difficult to detect. At $\xi=0.29$ which very nearly corresponds to the oilflow reattachment point, the mean velocity clearly indicates that mean reattachment has taken place. Since the measurement stations were $1.27-\mathrm{cm}$ apart, it is difficult to assess how accurately the mean separation and reattachment point locations could be determined from mean velocity profile measurements. Unfortunately, at $\xi=-3.44$ (the mean separation point from oil-flow visualization), data were not taken closer than $0.6 \mathrm{~mm}$ from the wall. At the other stations,

$\ddagger$ As pointed out by the reviewer. 


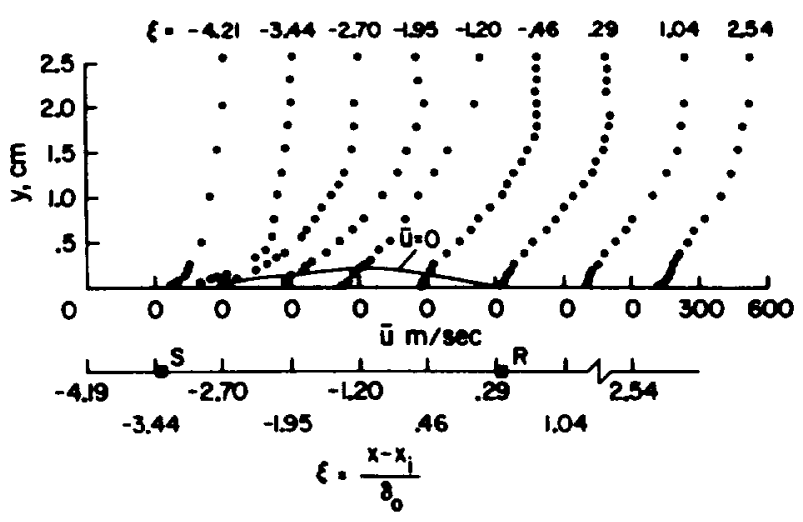

Fig. 8 Mean velocily profiles of the interaction region.

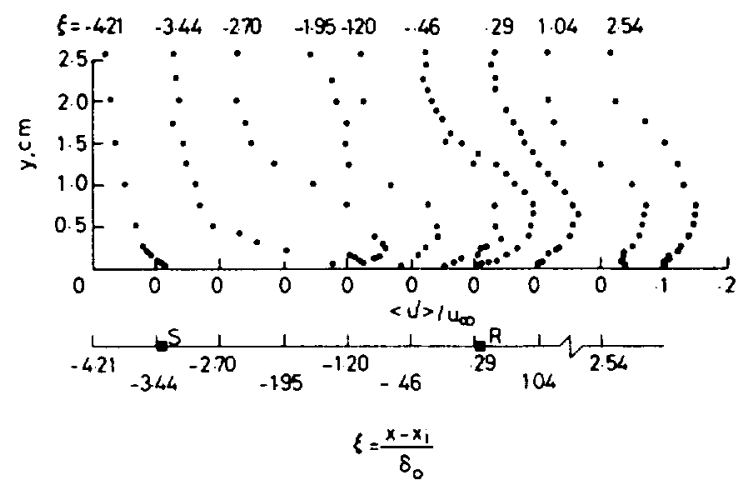

Fig. 9 Turbulence inlensity profiles of the interaction region.

measurements were obtained within $0.25 \mathrm{~mm}$ from the wall. The minimum velocity of $-65 \mathrm{~m} / \mathrm{s}$ was measured at the point, $\xi=-1.20 \mathrm{~cm}$ and $Y=0.1 \mathrm{~cm}$. Negative instantaneous velocities as low as $-200 \mathrm{~m} / \mathrm{s}$ were observed in the separated region. Included in Fig. 8 is the $\dot{u}=0$ line for the separated region.

The streamwise turbulence intensity profiles are shown in Fig. 9. At $\xi=-4.21$, the turbulence intensities near the wall were measured to be slightly higher than the corresponding values of the undisturbed profile. The turbulence intensity at the next downstream station $(\xi=-3.44)$ increased rapidly near the wall to the highest measured rms for the entire region $(32 \%)$. This turbulence was then diffused away from the wall as it moved downstream. The turbulence intensity near the wall at the subsequent downstream stations steadily decreased until reaching close to the initial wall value of $10 \%$ at $\xi=-$ 0.46 and then remained nearly constant. The maximum turbulence levels at these stations $(\xi \geq-0.46)$ also exhibited nearly the same value of $15 \%$. An unexpected observation was the dramatic decrease in turbulence levels between the $\xi=-1.20$ and -0.46 stations. At $\xi=-1.20$, the maximum turbulence intensity was $24 \%$, while at $\xi=-0.46$, it was only $15 \%$. It is near the $\xi=-1.95$ and -1.20 stations that the incident shock wave penetrates the boundary layer. This may explain the observed behavior. Due to shock motion, the measured rms at these two stations was substantially higher at the outer edge of the boundary layer, than at the other stations.

At each measurement station, a large number of instantaneous velocity readings were accumulated to form a histogram, from which the mean and rms velocities were calculated as described in the previous section. Four such histograms (probability density functions), which are representative of the histograms obtained, are illustrated in Fig. 10. The histograms are plotted with an arbitrary vertical scale (i.e., the areas under the distributions are not equal). A velocity histogram for a point outside the boundary layer is a)

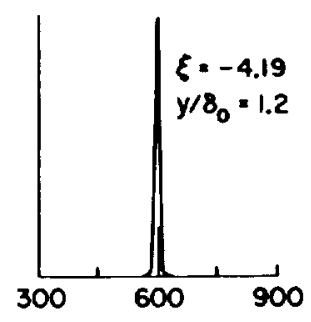

b)
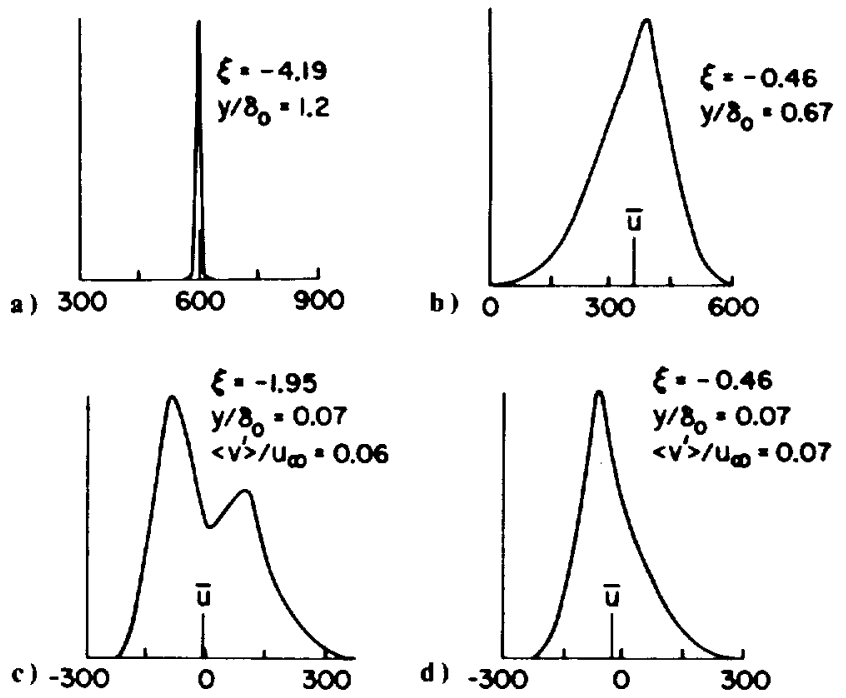

Fig. 10 Representative velocity histograms obtained in the interaction region.

given in Fig. 10a. Figure 10b gives the probability density function for the velocity data point within the boundary layer. The breadth of the histogram represents the degree of turbulence measured at this point. The histogram is slightly skewed toward the low velocities. Figure $10 \mathrm{c}$ is representative of the histograms which were obtained near the separation point close to the wall. It shows a "dip" near $u_{i}=0$. This unexpected character of the histograms was consistently observed at points near the mean separation point and as far downstream as $\xi=1.96$. Whereas, near the reattachment point, histograms like that shown in Fig. lod were consistently observed, Gaussian-type distributions with a slight skewness towards higher velocities and no dip at $u_{1}=0$. It will not be possible to explain fully the histograms without further experimental investigations. Nevertheless, some observations will be presented here:

1) Velocity biasing toward high-velocity particles as discussed in Ref. 12 can be the main cause of this behavior. Measurements of the velocity fluctuations normal to the wall $\left\langle v^{\prime}\right\rangle$ with the laser velocimeter revealed this quantity to be nearly the same at the separation and reattachment stations. Thus, for the observed behavior to be consistent with the arguments of Ref. 12, the correlation between the streamwise velocity fluctuations, $\left\langle u^{\prime}\right\rangle$ and the cross-stream velocity fluctuations, $\left\langle v^{\prime}\right\rangle$ would have to be markedly different at the separation and reattachment locations.

2) The dip in the histogram could be due to the two sizes of aerosols present in the flow. But this does not explain why the dip is always around $u_{i}=0$. The differences in the velocity distributions may be a result of bulk motion of the separation bubble caused by unsteadiness of the incident shock wave. This could only be true if one could show that the point of reattachment is insensitive to the jitter of the incident shock wave.

\section{Conclusions}

Laser velocimeter measurements were obtained for the flowfield produced by an oblique shock wave impinging on a turbulent boundary layer for Mach 2.9 freestream conditions. Boundary-layer data consisting of the mean velocity and turbulence intensity in the streamwise direction were realized from upstream of the interaction to approximately two boundary-layer thicknesses downstream of reattachment. A comparison of mean velocity profiles to pressure probe results demonstrated the superiority of the laser velocimeter technique in turbulent separated flows where the instantaneous velocity component in the streamwise direction changes sign with time. The indicated mean separation and reattachment points as determined from the mean velocity 
data were consistent with that predicted from oil-flow visualization studies. The minimum mean velocity measured in the separated region was $-65 \mathrm{~m} / \mathrm{s}$.

The turbulence intensity was observed to increase dramatically near the wall at the mean separation point, reaching the peak measured value of $32 \%$ relative to the freestream velocity. Further downstream, the turbulence appeared to be diffused across the boundary layer, with the peak value moving further from the wall with downstream distance. Near the wall, the turbulence level reached a nominal value of $10 \%$ just prior to the mean reattachment point, and maintained this level to the last measurement station. In the post-reattachment region, the peak turbulence intensity maintained a near constant level of $15 \%$.

The data were obtained using monodisperse latex particles of $0.5-\mu \mathrm{m}$ diam, which, based on response measurements across the incident oblique shock wave, were believed to provide satisfactory particle trackability. No corrections were applied to the data for the "velocity biasing" effect described in Ref. 12. If corrections were in order, this was not apparent from the data. Overall, the measurements were observed to be wholly consistent with the physics of the flow.

Differences observed in the velocity histograms near separation and reattachment may have some important bearing on the character of the flow. Near separation, a dip at $u_{i}=0$ was consistently recorded in the histograms, whereas near reattachment, this was not observed. The reason for this behavior is not fully understood. It does not appear to be explainable on the basis of a "velocity biasing" argument .

Based on the self-consistency of the measurements, the agreement with pressure probe results (where they could be considered reliable), and the agreement with oil-flow visualization results, it is believed that the data presented represent a good description of the mean-flow and turbulent behavior within the interaction zone of an oblique shock wave and a supersonic turbulent boundary layer.

\section{References}

'Wilcox, D. C., "Calculation of Turbulent Boundary Layer Shock Wave Interaction," AIAA Journal, Vol. 11, Nov. 1973, pp. 15921594.
${ }^{2}$ Baldwin, B. S. and MacCormack, R. W., “Numerical Solution of the Interaction of a Strong Shock Wave with a Hypersonic Turbulent Boundary Layer," AIAA Paper 74-558, 1974.

${ }^{3}$ Horstman, C. C., Kussoy, M. I., Coakley, T. J., Rubesin, M. M., and Marvin, J. G., "Shock-Wave-Induced Turbulent BoundaryLayer Separation at Hypersonic Speeds," AIAA Paper 753, 1975.

Baldwin, B. S. and Rose, W. C., "Calculation of ShockSeparated Turbulent Boundary Layers," NASA Conference on Aerodynamic Analyses Requiring Advanced Computers, Langley Research Center, Hampton, Va., March 4-6, 1975.

${ }^{5}$ Murphy, J. D., Presley, L. L., and Rose, W. C., "On the Calculation of Supersonic Separating and Altaching Flows," NASA Conference on Aerodynamic Analysis Requiring Advanced Computers, Langley Research Center, Hampton, Va., March 4-6, 1975.

${ }^{6}$ Goldstein, R. J., "Measurement of Fluid Velocity by LaserDoppler Techniques," Applied Mechanics Reviews, Vol. 27, June 1974, pp. 753-760.

${ }^{7}$ Eggins, P. L. and Jackson, D. A., "Laser Doppler Velocity Measurements in a Supersonic Flow Without Artificial Seeding," Physics Letters, Vol. 42A, Nov. 1972, pp. 122-124.

Bohnson, D. A., "Turbulence Measurements in a Mach 2.9 Boundary Layer Using Laser Velocimetry," AIAA Journal, Vol. 12, May 1974, pp. 711-714.

"Yanta, W. J. and Lee, R. E., "Determination of Turbulence Transport Properties with the Laser Doppler Velocimeter and Conventional Time-Averaged Mean Flow Measurements at Mach 3," AlAA Paper 74-575, Palo Alto, Calif., 1974.

${ }^{10}$ Johnson, D. A. and Rose, W. C., "Laser Velocimeter and HotWire Anemometer Comparison in a Supersonic Boundary Layer," AIAA Journal, Vol. 13, April 1975, pp. 512-515.

"Rose, W. C. and Johnson, D. A., "Turbulence in a Shock-Wave Boundary Layer Interaction," AIAA Journal, Vol. 13, July 1975, pp. 884-889.

${ }^{12}$ McLaughlin, D. K. and Teiderman, W. G., "Biasing Correction for Individual Realization of Laser Anemometer Measurements in Turbulent Flows," Physics of Fluids, Vol. 16, Dec. 1973, pp. 20822088.

${ }_{13}$ Reda, D. C. and Murphy, J. D., "Shock Wave-Induced Boundary-Layer Interactions in Rectangular Channels," $A / A A$ Journal, Vol. 11, Oct. 1973, pp. 1367-1368.

${ }^{14}$ Reda, D. C. and Murphy, J. D., "Shock Wave-Turbulent Boundary-Layer Interactions in Rectangular Channels, Part II: The Influence of Sidewall Boundary Layers on Incipient Separation and Scale of the Interaction," AlAA Paper 73-234, Washington, D.C., 1973; also AIAA Journal, Vol. 11, Oct. 1973, pp. 1367-1368. 